\title{
Psychosocial Indicators via Hand Tremor
}

\author{
Ted Selker, Patricia Collins, and Will Dayton \\ Carnegie Mellon University Silicon Valley, Moffett Field, CA 94035 \\ \{ted.selker, patricia.collins\}@sv.cmu.edu, wdayton@gmail.com
}

\begin{abstract}
We propose hand tremor as a new type of input that can corroborate psychosocial conditions. An Android application was able to distinguish tremor variability differences between people with and without diagnosed hand tremor. Tremor measurements also corroborated self-assessment of sleep quality. Hand tremor evaluation may be a monitorable, implicit input to systems that respond to various psychosocial states. We encourage researchers to consider how interface design changes when using implicit sensors such as tremor sensing.
\end{abstract}

Keywords: psychosocial indicators, novel user interface, hand tremor, implicit input.

\section{Introduction}

Tremor, an involuntary, semi-periodic movement, is the most common movement disorder [4]. Most people exhibit physiologic hand tremor, a benign, low-amplitude variation in neuromuscular state [5]. Tremor may be heightened by many factors such as strong emotion, physical exhaustion, hypoglycemia, hyperthyroidism, stimulants, alcohol intake or withdrawal, or fever [1]. Other factors such as sleep [2] and smoking [7] have been shown to affect tremor significantly.

With a growing number of smartphones sold annually [3], accelerometers provide an omnipresent opportunity to measure the state of hand tremor.

Previous efforts to measure tremor to improve cellphone user interfaces have been unsuccessful. Quek, for example was not able to measure body tremor reliably to recognize when a phone was being held [8]. Other attempts at improving the user's experience by reducing the impact of tremor have had limited success [4].

The presented research demonstrates that even if tremor status might not completely indicate the user's condition, it might be part of sensor and contextual information fusion that reveals psychosocial state. The system could then provide context-specific guidance or feedback to the user.

\section{Method}

Most hand tremor is in the 3-6 Hz range, although certain types can reach $12 \mathrm{~Hz}$ [6]. We developed an application that detects tremor up to 19 HZ the HTC G1 phone, running Android 1.6. The mobile phone application records 3-D accelerometer while the subject holds the phone with an unsupported arm for 30 seconds. The number of amplitude maximums; tremor frequency; amplitude average, minimum, and 
maximum; period average, minimum, maximum; and standard deviation of period are stored on a micro SD card.

A pilot study included volunteers with and without tremor disorder. For each measurement, the phone's display first instructs a user to "hold the phone comfortably in your dominant hand with the screen facing you. As long as you comfortably can, extend your arm fully while keeping it perpendicular to your body. Remain as still as possible for the duration of the test."

A formal study was conducted (age $\geq 65$ ) at a senior center. Subjects filled out a pretest survey of experience with hand tremor, took tremor measurements, and filled out the Pittsburgh Sleep Quality Index (PSQI) [9]. Subjects were given the option to have the experimenter read the sleep survey aloud and to record their responses.

\section{Results}

The pilot study included one subject with diagnosed tremor used the application for a few days. Several times each day this subject took a measurement, noting no tremor ( $\mathrm{N}=8$ times), slight tremor ( $\mathrm{N}=4$ times), or clearly visible tremor ( $\mathrm{N}=7$ times). Analysis easily distinguished between the three reported states (figure 1 and 2).

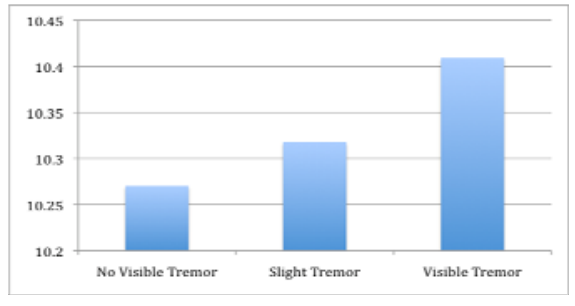

Fig. 1. Mean amplitude maximum $\left(\mathrm{m} / \mathrm{s}^{2}\right)$

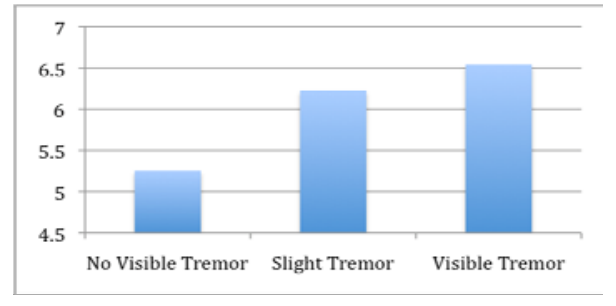

Fig. 2. Mean Tremor Frequency (Hz)

Unlike amplitude, frequency clearly distinguished the subject with known tremor. None of the non-tremor pilot subjects recorded above $5 \mathrm{~Hz}$, lower than the tremor subject's mean "no visible tremor." Most subjects' hand movement was in the 3-4 Hz range. Standard deviation of period also proved to distinguish between- and withinsubject comparisons (figure 3). No other pilot subject recorded a standard deviation as low as the tremor subject's.

The pretest survey and data collection protocol were administered to 19 formal study subjects interacting with a phone as a tremor sensor. Sixteen completed the PSQI sleep survey as well. Five reported a history of hand tremor disorder. As in the pilot experiment, amplitude profiles did not distinguish between tremor and no-tremor subjects (Table 1). However, the two groups on frequency measurements yields a tscore of 5.06 for one degree of freedom with less than 0.1 of the result occurring by chance. Comparing the two groups on mean period standard deviation yields a tscore of 3.70 , less than 0.1 that the result occurred by chance. As with pilot results, the formal study results suggest that those with hand tremor have higher frequency of tremor and lower standard deviation. 


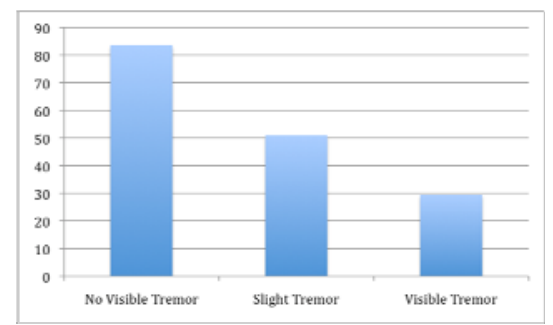

Fig. 3. Mean standard deviation tremor time period (milliseconds)

The sleep index graph (figure 4) shows a nearly linear correlation with hand tremor frequency. A correlation analysis of PSQI scores and tremor frequency yields an r-score of 0.557 with a probability of less than 0.05 that the result occurred by chance.

Table 1. Tremor amplitude and frequency between diagnosed tremor and no tremor groups

\begin{tabular}{|c|c|}
\hline $\begin{array}{c}\text { No History of } \\
\text { Hand Tremor } \mathrm{N}=14\end{array}$ & $\begin{array}{l}\text { History of Hand } \\
\text { Tremor: } \mathrm{N}=5\end{array}$ \\
\hline \multicolumn{2}{|c|}{ Mean Amplitude Maximum (m/ $\left./ \mathbf{s}^{2}\right)$} \\
\hline 10.28 & 10.30 \\
\hline \multicolumn{2}{|c|}{ Mean Frequency (Hz): t-score 5.06} \\
\hline 3.52 & 4.95 \\
\hline \multicolumn{2}{|c|}{$\begin{array}{l}\text { Mean Period Standard Deviation (ms): } \\
\text { t-score 3.70 }\end{array}$} \\
\hline 138.29 & 77.11 \\
\hline
\end{tabular}

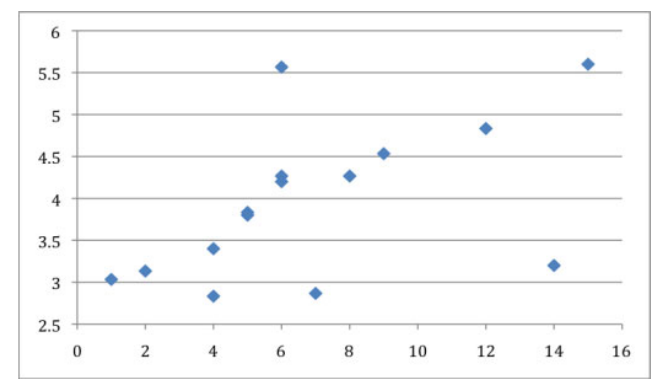

Fig. 4. Scatter plot of PSQI Scores (x-axis) and hand tremor frequency ( $\mathrm{y}$-axis)

\section{Discussion}

Smartphone measurements of tremor frequency corroborated self-report of sleep quality and tremor. The use of such an application to address the healthcare needs of those with tremor appears feasible. We report on overcoming earlier reported obstacles to using tremor to recognize tremor presence [8] and go on to distinguish diagnosed tremor and sleep quality in tremor period and standard deviation .

We examined the single factor of sleep quality and found a positive correlation between the PSQI and hand tremor. Tremor is also associated with smoking, caffeine use, physiological and emotional stress--all factors that could be examined to allow users to gain a better understanding of their health.

Our frequency domain instrument consistently distinguished tremor in subjects ranging from their 20s into their 80s. This instrument supports comparisons across people without calibration. This work only begins the story of what might be done with indirect indicators like hand tremor. 


\section{Conclusion and Future Work}

The frequency-domain smartphone instrument could simplify neurological diagnosis and give more accurate and responsive ways of prescribing and adjusting medication. Further study should examine fidelity of the measurements and how to present data for decision-making and feedback.

Tremor may be heightened by many factors. The specific factor (e.g., hypoglycemia) and the tremor state could be used to provide timely information or guidance to the user (e.g., early warning of a hypoglycemic state). Tremor is the kind of subtle input that can take user experience forward into the psychosocial realms of personal experience and interpersonal exchanges. Tremor is an example of a new kind of input that can add to or corroborate other sensory inputs and might be used in reflective- as well as directive-style interfaces.

\section{References}

1. Tremor Fact Sheet. National Institute of Neurological Disorders and Stroke, National Institutes of Health,

http: //www.ninds.nih.gov/disorders/tremor/detail_tremor.htm

2. The Causes and Effects of Lack of Sleep, Sleep Deprivation. Sleep-Deprivation.com, June 2 (2010), http://www.sleep-deprivation.com/articles/types-ofsleep-disorder/lack-of-sleep/index.php

3. Park, W.: Apple Boosts Global Smartphone Market Share Big-Time, Holds on to No. 3 Position. Into Mobile, May 7 (2010),

http: / /www. intomobile.com/2010/05/07/apple-boosts-global-

Smartphone-market-share-big-time-holds-on-to-no-3position.html

4. Smaga, S.: Tremor. American Family Physician 68(8), 1545-1552 (2003)

5. Strachan, S., Murray-Smith, R.: Muscle tremor as an input mechanism. In: UIST 2004 (2004)

6. Zesiewicz, T.A., Hauser, R.A.: Phenomenology and treatment of tremor disorders. Neurol. Clin. 19, 651.vii-680.vii (2001)

7. Maykoski, K.A., Rubin, M.B., Day, A.C.: Effect of Cigarette Smoking on Postural Muscle Tremor. Nursing Research 12, 39-43 (1997)

8. Quek, M.: Background sensing using a mobile device. Diss., University of Glasgow (2008)

9. Buysse, D.J., Reynolds, C., Monk, T., et al.: Pittsburgh Sleep Quality Index: a new instrument for psychiatric practice and research. Psychiatry Res. 28, 193-213 (1989a) 